\title{
Magnetic coupling to the advanced Virgo payloads and its impact on the low frequency sensitivity
}

A. Cirone, A. Chincarini, M. Neri, S. Farinon, G. Gemme, I. Fiori, F. Paoletti, E. Majorana, P. Puppo, P. Rapagnani, P. Ruggi, and B. L. Swinkels

Citation: Review of Scientific Instruments 89, 114501 (2018); doi: 10.1063/1.5045397

View online: https://doi.org/10.1063/1.5045397

View Table of Contents: http://aip.scitation.org/toc/rsi/89/11

Published by the American Institute of Physics

\section{Articles you may be interested in}

Drop hammer with high-speed thermal imaging

Review of Scientific Instruments 89, 115104 (2018); 10.1063/1.5051357

Note: An automatic liquid nitrogen refilling system for small (detector) Dewar vessels

Review of Scientific Instruments 89, 116101 (2018); 10.1063/1.5046637

Record indoor magnetic field of $1200 \mathrm{~T}$ generated by electromagnetic flux-compression

Review of Scientific Instruments 89, 095106 (2018); 10.1063/1.5044557

Invited Article: Advances in tunable laser-based radiometric calibration applications at the National Institute of Standards and Technology, USA

Review of Scientific Instruments 89, 091301 (2018); 10.1063/1.5004810

Single-shot frequency-resolved optical gating for retrieving the pulse shape of high energy picosecond pulses Review of Scientific Instruments 89, 103509 (2018); 10.1063/1.5044526

Cavity ring-down spectroscopy to measure negative ion density in a helicon plasma source for fusion neutral beams

Review of Scientific Instruments 89, 103504 (2018); 10.1063/1.5044504

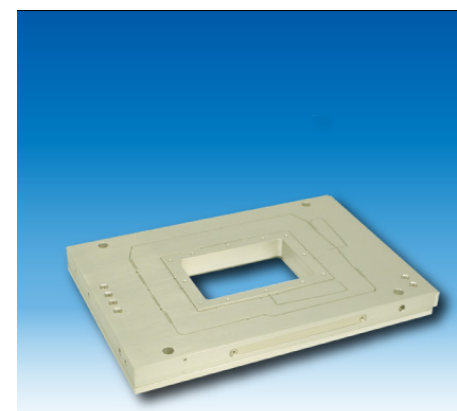

Nanopositioning Systems

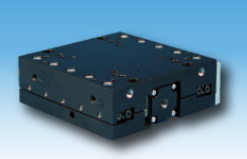

Micropositioning

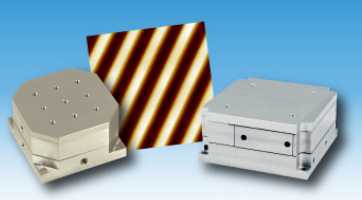

AFM \& SPM

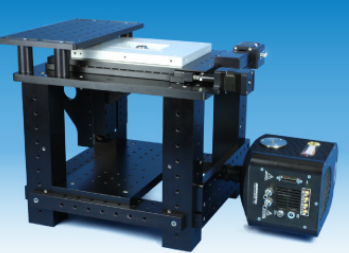

Single molecule imaging 


\title{
Magnetic coupling to the advanced Virgo payloads and its impact on the low frequency sensitivity
}

\author{
A. Cirone, ${ }^{1,2, a)}$ A. Chincarini, ${ }^{1}$ M. Neri, ${ }^{2}$ S. Farinon, ${ }^{1}$ G. Gemme, ${ }^{1}$ I. Fiori, ${ }^{3}$ F. Paoletti, ${ }^{4}$ \\ E. Majorana, ${ }^{5}$ P. Puppo, ${ }^{5}$ P. Rapagnani, ${ }^{5,6}$ P. Ruggi, ${ }^{3}$ and B. L. Swinkels ${ }^{7}$ \\ ${ }^{1}$ INFN, Sezione di Genova, I-16146 Genova, Italy \\ ${ }^{2}$ Università degli Studi di Genova, I-16146 Genova, Italy \\ ${ }^{3}$ European Gravitational Observatory (EGO), I-56021 Cascina, Pisa, Italy \\ ${ }^{4}$ INFN, Sezione di Pisa, I-56127 Pisa, Italy \\ ${ }^{5}$ INFN, Sezione di Roma, I-00185 Roma, Italy \\ ${ }^{6}$ Università di Roma "La Sapienza," I-00185 Roma, Italy \\ ${ }^{7}$ Nikhef, Science Park 105, 1098 XG Amsterdam, The Netherlands
}

(Received 20 June 2018; accepted 9 October 2018; published online 1 November 2018)

\begin{abstract}
We study the electromagnetic coupling of the Advanced Virgo (AdV) input mirror payload in response to a slowly time-varying magnetic field. As the problem is not amenable to analytical solution, we employ and validate a finite element (FE) analysis approach. The FE model is built to represent as faithfully as possible the real object, and it has been validated by comparison with experimental measurements. The intent is to estimate the induced currents and the magnetic field in the neighbourhood of the payload. The procedure found 21 equivalent electrical configurations that are compatible with the measurements. These have been used to compute the magnetic noise contribution to the total AdV strain noise. At the current stage of development, AdV seems to be unaffected by magnetic noise, but we foresee a non-negligible coupling once AdV reaches the design sensitivity. Published by AIP Publishing. https://doi.org/10.1063/1.5045397
\end{abstract}

\section{INTRODUCTION}

The Advanced Virgo (AdV) experiment, ${ }^{1,2}$ hosted by the European Gravitational Observatory (EGO) in Cascina (Pisa), is a Michelson-like laser interferometer endowed with two 3 $\mathrm{km}$ long Fabry-Perot resonant cavities and 4 suspended mirror test masses. Its purpose is the detection of Gravitational-Waves (GW) of astrophysical and cosmological origin.

The first detection occurred in September 14, 2015, ${ }^{3}$ when a transient signal produced by the coalescence of two stellar mass black holes was pinpointed from the two advanced Laser Interferometer Gravitational-Wave Observatory (aLIGO) detectors. ${ }^{4}$ From that moment on, several other events were observed. ${ }^{5-7}$

At this stage, AdV can observe a volume of universe 30 times bigger than that accessible to Virgo+, which was the previous detector configuration. ${ }^{8}$ This was made possible by increasing the sensitivity, with the drawback that new noise sources became relevant. In the range of frequency of 10 $100 \mathrm{~Hz}$, one of the limiting noises could be due to the magnetic coupling through the coil-magnet pairs used as actuators in payloads, which are the mechanical assemblies that suspend the test masses and other ancillary components, including the actuation devices.

This kind of coupling was already observed during the first Virgo Scientific Run (2008), when the substitution of the magnets with five-times smaller ones reduced the magnetic noise contribution to the sensitivity. ${ }^{9}$

\footnotetext{
a) Author to whom correspondence should be addressed: alessio.cirone@ ge.infn.it
}

Magnetic coupling can also account for the correlated magnetic noise from Schumann resonances, which threatens to contaminate the observation of a Stochastic GravitationalWave Background (SGWB) in interferometric detectors. ${ }^{10,11}$

In addition, magnetic field transients could also enter the analysis pipeline so that the magnetic coupling can also affect searches for transient GW signals, as reported in Ref. 12.

The first estimation of the magnetic coupling effect had a large uncertainty, ${ }^{13}$ and therefore the overall magnetic noise issue is still open.

In this work, we study the magnetic coupling and its impact on the detector sensitivity. We tackle this problem both with Finite Element (FE) simulations and with measurements of the magnetic response of a complex conductive object (the payload), surrounded by a slowly time-varying magnetic field.

Our goal is to determine the magnetic field around a complex, composite object, for given external magnetic field conditions. For that, we need to estimate the detailed electrical configuration and the eddy current flow, in a situation where we lack a reference standard and the analytical solution is not trivial.

The procedure consists of 3 steps: the construction of the FE model (Sec. IV), its validation (Sec. V), and the magnetic response estimation in the Virgo environment (Sec. VI).

\section{THE ADVANCED VIRGO PAYLOADS}

The AdV payload (PAY) consists of two suspension stages: the marionette, which is a structure holding the test mass (TM) and the actuator cage. ${ }^{14}$ The PAY is suspended to the last stage of the so-called Virgo superattenuator, which is 

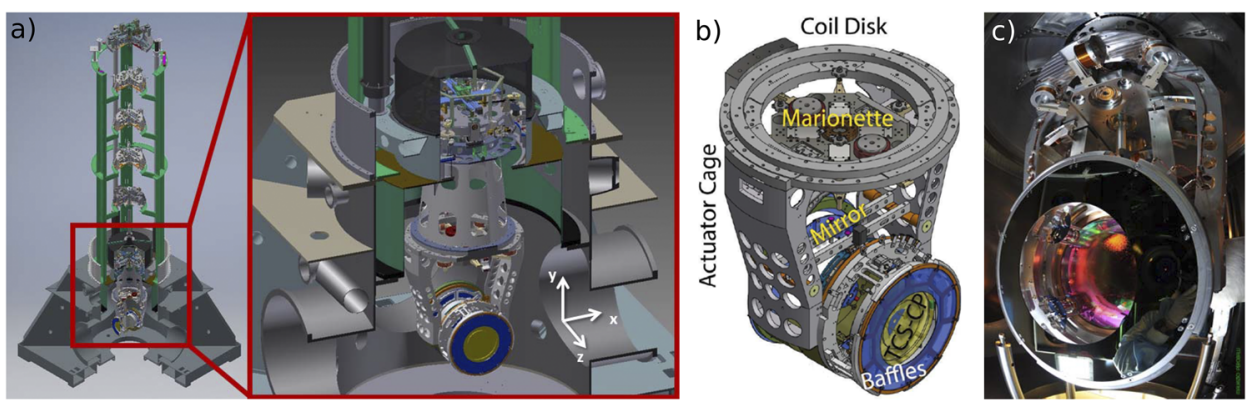

FIG. 1. (a) CAD (Computer-Aided Design) drawings of the AdV Input Mirror Payload (IMP) integrated in the approximately $10 \mathrm{~m}$ superattenuator suspension tower; (b) CAD drawing of the IMP, including the main metallic assemblies surrounding the TM; (c) photo of the integrated IMP. a series of six vertical and six horizontal passive mechanical filters [Fig. 1(a)]. The overall system is designed to suppress the seismic vibrations by many orders of magnitude, starting from a few Hz. ${ }^{15}$ The typical configuration of the PAY is shown in Fig. 1(b). The main structures close to the magnets are the actuator cage, the marionette, and a set of ring-shaped components surrounding the TM (baffles, ring heater, Compensation Plates-CP, etc...). The cage is directly connected to the last stage of the superattenuator through the coil disk. It also supports the set of driving coils that act on a total of 8 permanent magnets $\left(\mathrm{Sm}_{2} \mathrm{Co}_{17}\right.$ magnets of $1 \mathrm{~T}, 8 \mathrm{~mm}$ in diameter, and $4 \mathrm{~mm}$ thick) glued on the marionette. ${ }^{2}$ The magnetic mount of the 8 actuators has horizontal and vertical orientations, in an anti-parallel configuration [Fig. 2(a)]. This coil-magnet system steers the PAY in three degrees of freedom: the translation along the beam (roll) and the rotations (pitch and yaw) around the other two orthogonal axes. Starting from this general structure, each suspension chain is optimized in different ways so that we have 4 different types
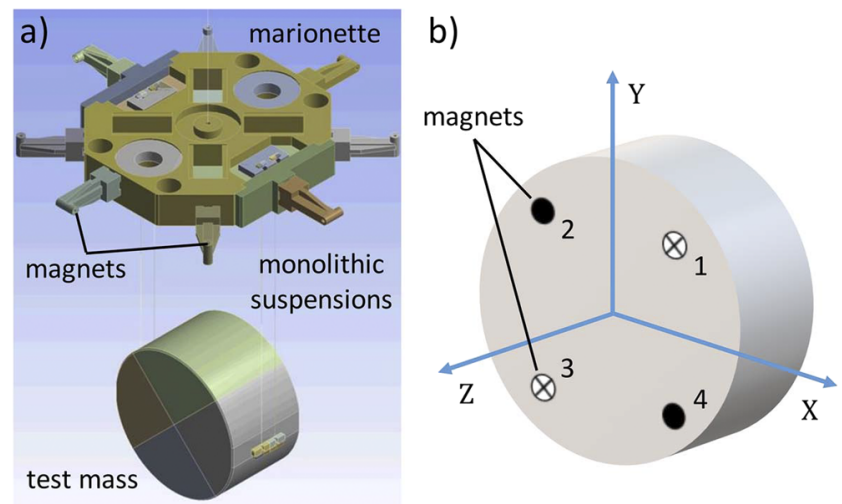

FIG. 2. (a) Schematics of the last suspension stage of the TM, which includes the marionette with 8 actuation magnets, 4 fused silica suspension fibres, and the TM itself; (b) schematic view of the cross anti-parallel configuration of the four magnets glued on the TM.

TABLE I. Properties of the magnets glued on the surface of each TM.

\begin{tabular}{lc}
\hline \hline Properties & Values \\
\hline Material & $\mathrm{Sm}_{2} \mathrm{Co}_{17}$ \\
Diameter $(\mathrm{mm})$ & $1.5 \pm 0.1$ \\
Thickness $(\mathrm{mm})$ & $1.5 \pm 0.1$ \\
Residual induction $(\mathrm{T})$ & $1.0 \pm 0.15$ \\
Magnetic moment $\left(\mathrm{mA} \times \mathrm{m}^{2}\right)$ & $2.0 \pm 0.3$ \\
\hline
\end{tabular}

of PAY [e.g., the Input Mirror Payload-IMP—in Fig. 1(c)]. Other four coil-magnet pairs act directly on the TM, with the magnets (properties in Table I) glued in a cross anti-parallel configuration [Fig. 2(b)]. This solution is expected to be the most effective against electromagnetic disturbances.

Indeed these magnets are sensitive not only to the magnetic field produced by the driving coils but also to any (noisy) external magnetic gradient. While the anti-parallel configuration of all the magnets located on both marionette and TM should be insensitive to any contribution that is spatially uniform, asymmetries due to a distinctive field-structure can produce a net total force, causing a displacement noise. In addition, the magnetic forces act on the TM directly, bypassing all the seismic isolation provided by the suspension system.

\section{MAGNETIC COUPLING TO THE PAYLOADS}

Magnetic disturbances can be expressed as a superposition of different magnetic contributions derived both from known point-like sources (local) and unknown ones (environmental). In the estimation of the magnetic noise, we considered only environmental components because it is assumed that there are no nearby magnetic sources. Under this hypothesis, the contribution of this noise to the AdV noise budget is relevant only in the low frequency range because at high frequencies (roughly above $150 \mathrm{~Hz}$ ) the interferometer sensitivity is dominated by other contributions, while the environmental magnetic field (at the PAY location) is filtered out by the metallic enclosure which surrounds the PAY. In the noise budget estimation, we explicitly consider the low-pass filtering effect of the steel tank.

A magnetic field acts on a permanent magnet causing the following two effects: (i) translational force associated with the magnetic gradient: $\mathbf{F}=\nabla(\boldsymbol{\mu} \cdot \mathbf{B})$, where $\mu$ is the magnet's magnetic moment, and (ii) torque produced by the magnetic force: $\tau=\mathbf{F} \times \mathbf{r}$. Actually we should talk about two distinct components of torque: one related to the TM and the other related to each single magnet. However, in the point-like magnet approximation, we neglect the second contribution, especially because the magnets are glued on the TM and the whole system is treated as a rigid body.

In advanced Virgo, all the magnets used for the TM actuation have approximately the same magnetic moment, directed along the beam propagation direction (i.e., $z$ ). The force on each magnet can be written as $F_{z}=\mu(\partial B / \partial z)$, so the total force on the TM is simply the sum of the forces on the four magnets. 
In the ideal situation in which the four magnets have exactly the same magnetic moment and if the magnetic field gradient is the same on each magnet, the anti-parallel cross configuration should guarantee a null force on the TM. In the real-world case, $\boldsymbol{\mu}$ has a tolerance of about $15 \%$ around the nominal value and the magnetic field gradient spatial symmetry is not guaranteed.

The main cause for a non-uniform gradient is the interaction between the external field and the metallic structure of the PAY. This structure couples with any time-varying magnetic field generating eddy currents, which in turn warp the field and produce a gradient.

We study only the IMP because-together with the End Mirror Payload (EMP) - it is the most sensitive to magnetic coupling.

The study of the magnetic response of an object is a classical electromagnetic problem where analytical solutions exist only for very simple geometries. If the goal is to know the magnetic field in each point of a volume, direct measurements are also impractical. The typical approach is to use a numerical solution [i.e., Finite Element (FE) analysis].

\section{SYSTEM MODELING}

The PAYs are very complex objects consisting of several parts (ten main parts; volume of about $1.2 \mathrm{~m}^{3}$ ) of different materials that are assembled by bolts, welds, or screws. We chose to use the COMSOL Multiphysics FE analysis simulation software ${ }^{16}$ and the AC-DC module for the computation of our time-varying magnetic field studies. For a closer look into some delicate simulation steps (e.g., geometry simplification and model meshing), see the supplementary material (Sec. I).

A subtle but significant problem lies in how parts are assembled. When simulating two adjacent metallic domains (i.e., in close physical contact), the software implicitly assumes electrical conductivity between them. In practice though, when two metallic parts are assembled without welding, the electrical contact can be impaired by a thin insulating film (e.g., oxides, contaminants, reaction products), which introduces an energy barrier that can limit the current flow. ${ }^{17,18}$ Depending on the thickness of the barrier, electrons may not have enough energy to tunnel across it. In particular, eddy currents are very feeble and therefore they are especially susceptible to energy barriers. The conductivity across two surfaces in electric contact is determined by several factors: the type of material, the surface finishing, and the pressure applied between the two surfaces. We designed and validated a simple model (open coil) which verified that the eddy currents flow critically depends on the force applied to keep two aluminum surfaces in contact. We experimentally measured a sharp transition of the eddy currents with respect to the force applied. This simple experiment prompted us to consider each connection among the metallic parts of a composite object as a two-state system (open-closed) in order to take into account the (unknown) state of the surfaces and the applied pressure.

The subdivision of a complex object such as the PAY into disjointed parts can be parametrized by the conductivity at
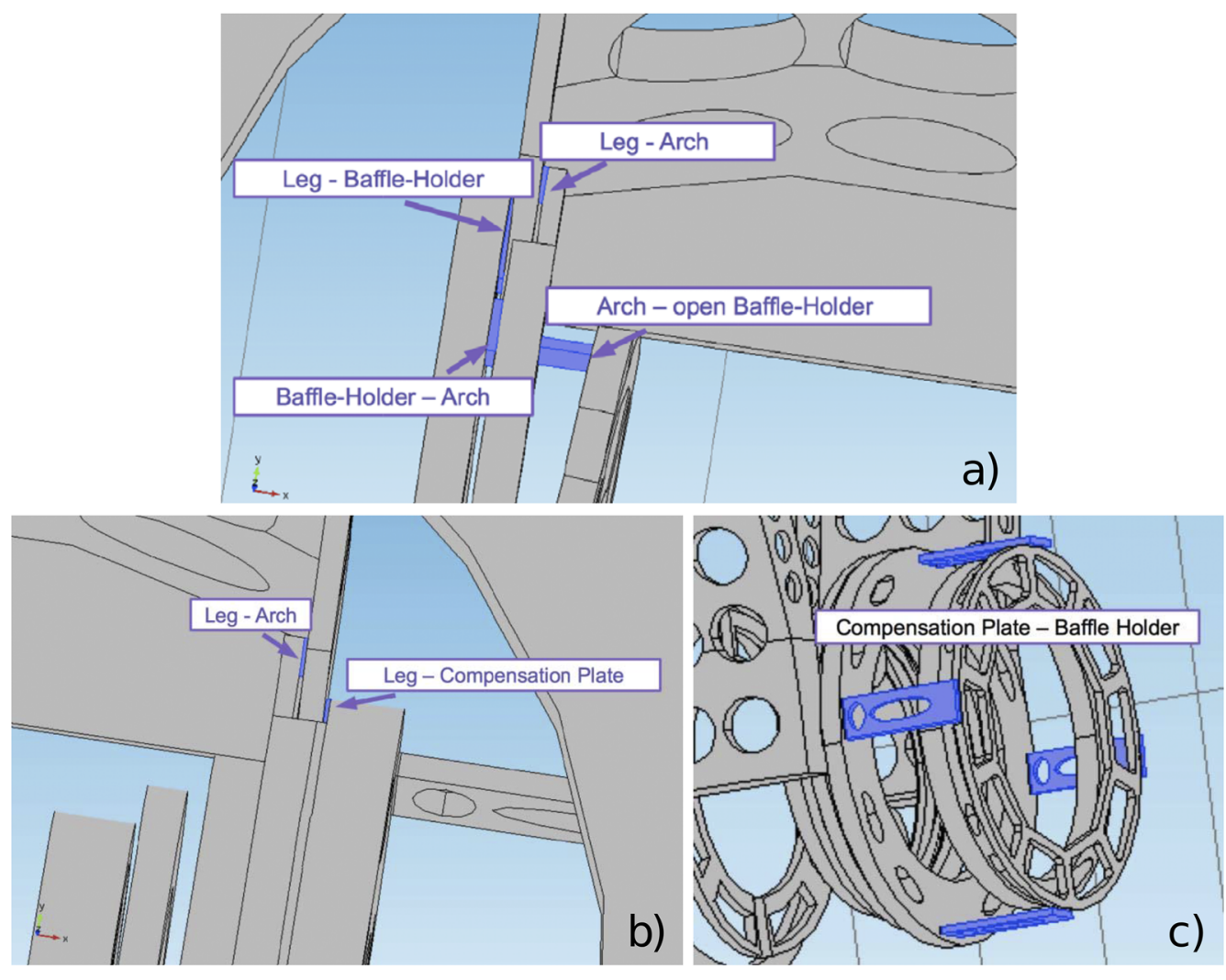

FIG. 3. Mechanical connections used to simulate a two-state electrical system: (a) Cage legs-baffle holder, cage legs-arch, arch-baffle holder and arch-open baffle holder; (b) cage legs-compensation plate, and cage legs-arch; (c) compensation plate-baffle holder. 
the level of each mechanical connection $p$ as 1 or 0 , leading to a very large number of configurations $\left(2^{p}\right)$. Therefore we employed a hierarchical analysis to capture only the most relevant contact points in the PAY assembly.

Overall, we found $p=7$ main connections which have a relevant impact on the gradient nearby the magnets. The corresponding parametrized volumes were inserted in correspondence with the real mechanical connections (screws and bolts), as presented in Fig. 3, and they were modelled in COMSOL using either air (insulation) or aluminum (the same material as the bulk).

The PAY assembly is made of different materials: aluminum, steel, and titanium. In the FE analysis, we modelled only the aluminum components, as they are the most relevant ones in terms of the number of parts and they have the highest conductivity, $3.03 \times 10^{7} \mathrm{~S} / \mathrm{m}$, compared to $1.4 \times 10^{6} \mathrm{~S} / \mathrm{m}$ for steel and $0.6 \times 10^{6} \mathrm{~S} / \mathrm{m}$ for titanium.

The model was embedded in a uniform, sinusoidal magnetic field of frequency $f=\omega / 2 \pi$.

\section{MODEL VALIDATION}

The FE model delivers the electromagnetic field at any point, but we still have to determine the electrical connection states among the relevant parts of the assembly (model validation). This means that we have to find out which of the $2^{7}=128$ possible models represents the real PAY at best, that is, what we need to compare each FE simulation with an experimental measure. In addition, we can use the measures to tune the geometrical representation and the uncertainties on the material properties.

The validation procedure requires an accurate setup, where the driving magnetic field and the geometrical properties are well under control, so that discrepancies between the simulation and the measurements can be assigned to the model assumptions.

\section{A. Experimental setup}

We built a driving system with two coils ("Big" and "Small," with an external radius of, respectively, 1.040 $\pm 0.001 \mathrm{~m}$ and $0.540 \pm 0.001 \mathrm{~m}$ ) and a structural PVC cubic frame that aligns them with the PAY (Fig. 4). The coils are made of 100 turns of copper wire with a diameter of 0.95 $\pm 0.02 \mathrm{~mm}$. The PAY was housed between the coils and two gridded panels with several reading slots, where we can insert a magnetic probe: this is a triaxial magnetic field sensor FL3-100 (Stefan Mayer Instruments), with intrinsic noise $<20 \mathrm{pT} / \sqrt{\mathrm{Hz}}$ at $1 \mathrm{~Hz}$ and a measurement range of $\pm 100 \mu \mathrm{T}$. The slots are geometrically tailored to the probe in order to minimize positional errors. They are designed to get a fairly dense sampling of the magnetic field. Some of the holes are bigger so that a probeholder rod can be inserted, in order to make spot measurements along a line inside the volume of the PAY. A picture is provided as the supplementary material (Fig. S1). The two coils generate spatially different field configurations and therefore provide a more accurate validation. They can be independently driven by an AC current generator (CoCo80, Crystal Instruments) coupled to a linear amplifier (BAA 120, TIRA). The cubic frame was placed in the Virgo Central Building (CEB), in a class 100 clean room under the input towers. We ensured that there were no significant metallic objects in a radius of $\approx 2 \mathrm{~m}$ around the apparatus (Fig. S2 of the supplementary material).

\section{B. Reference measurements}

We began our validation with the "zero" measure, that is, a set of reference measurements to study the contribution of the surrounding environment and the cubic frame alignment.

The measurements were performed using the experimental setup without the PAY inside it. Occasionally the amplifier exhibited a small drift from the nominal value of $1 \mathrm{~A}$, which was taken into account in the post-processing analysis by performing a current optimization. The three components of the magnetic field were measured on a set of 65 holes for each panel.

At the same time, we simulated the structural frame and the coils. The PVC frame is transparent to magnetic fields, allowing us to consider only two materials in the simulation: copper for the coil windings and "air" for the remaining parts. The magnetic field was calculated in correspondence with the measurement points in all the explored configurations. Finally we compared the experimental and simulated data by minimizing the relative difference function $m_{k}$, with $k=[1$, number of measuring points], between the measured and the simulated magnetic field value over the input current,

$$
m_{(k)}=\left|\frac{B_{\text {meas }}^{(k)}-c B_{\text {sim }}^{(k)}}{B_{\text {meas }}^{(k)}}\right|,
$$
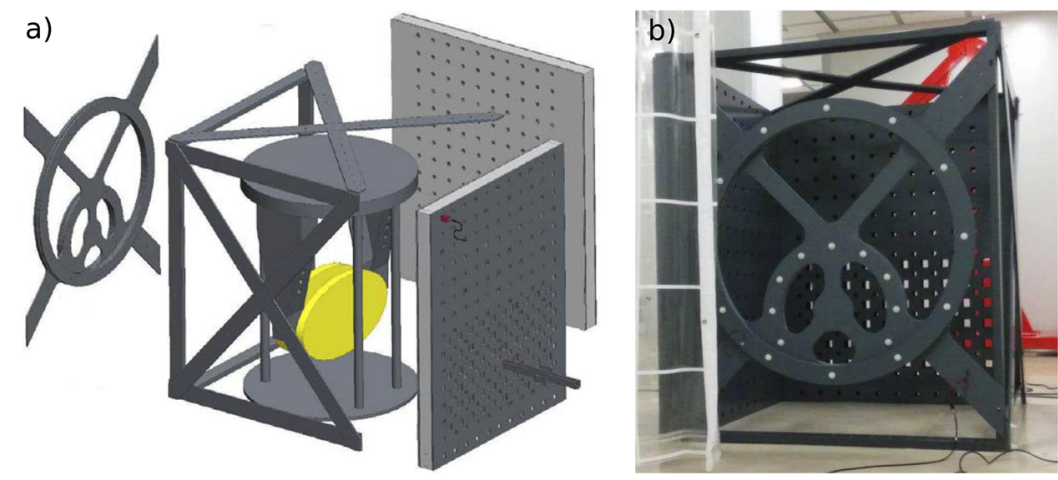

FIG. 4. The PVC frame built for the FEA validation: CAD drawing (a) and the actual object inside the Virgo clean room (b). The structure embeds two integrated coils on one side and two gridded panels on the opposite sides for accurate positioning of the magnetic probe. 


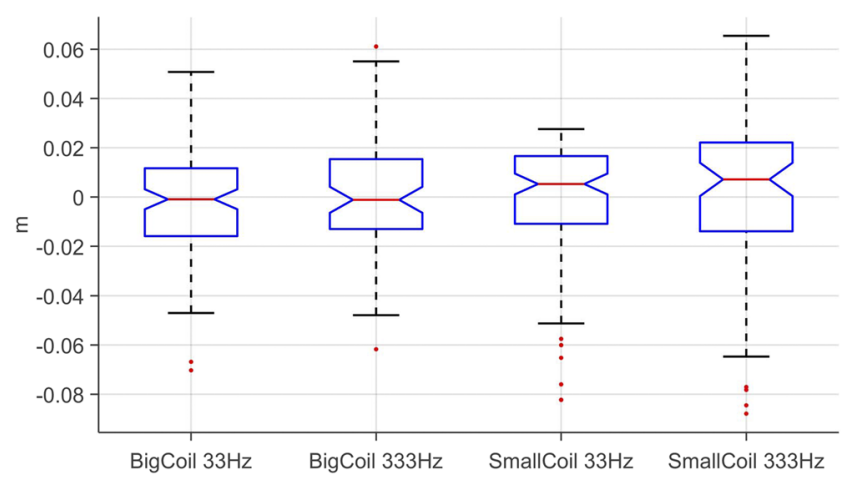

FIG. 5. Distributions of $m$ without PAY [Eq. (1), reference measure]. The statistics is computed on all the measured positions, for both coils and two frequencies. The red horizontal line is the median value, while the bottom and top edges of the blue boxes indicate the 25 th and 75 th percentiles, respectively. The whiskers extend up to 1.5 of the box range, and the outliers are plotted individually using single red dots.

where $c$ is the optimization parameter. Measurements and simulation were found to agree within $\approx 5 \%$, after the current optimization. Looking at the data (Fig. 5), we assess that the distribution of the relative differences is compatible with zero for both the "Big" and "Small" coil configurations and at both $33 \mathrm{~Hz}$ and $333 \mathrm{~Hz}$.

\section{Input mirror payload measurement}

The entire procedure was repeated with the IMP inside the frame. The PAY was kept in place by using a support structure with an aluminum base and four steel legs [Fig. 6(a)]. The PAY was laser-aligned with the coil axis (estimated accuracy of $\simeq 1 \mathrm{~cm}$ ), and measurements were taken both in correspondence with the two gridded panels and with the help of the extension rod, to get as close as possible to the PAY assembly.

The validation goal is to identify which electrical configuration best represents the real PAY. The procedure is detailed in the supplementary material (Sec. II).

Eventually, we identified 21 statistically equivalent configurations that can represent the real object: the equivalence was defined by using the non-parametric KolmogorovSmirnov test, applied to the sorted distribution versus the best one. Since all these configurations are statistically equivalent,
TABLE II. Mean and standard deviation of $\mathrm{m}$ [Eq. (1)] with and without the PAY structure. Values combine both frequencies $(73 \mathrm{~Hz}$ and $33 \mathrm{~Hz}$ ) and are given for the two source configurations ("Big" and "Small" coils).

\begin{tabular}{lc}
\hline \hline Configuration & $\left(B_{\text {meas }}-B_{\text {sim }}\right) / B_{\text {meas }}$ \\
\hline Payload-“small" coil & $-0.031 \pm 0.021$ \\
Payload-"big" coil & $-0.0057 \pm 0.0220$ \\
Reference-"small" coil & $0.0052 \pm 0.0090$ \\
Reference-"big" coil & $-0.00093 \pm 0.00740$ \\
\hline \hline
\end{tabular}

all of them have to be considered in the evaluation of the magnetic noise contribution to the advanced Virgo sensitivity. The agreement between measurements and simulations is presented in Table II, where also the reference case at $33 \mathrm{~Hz}$ is reported once more for comparison. These values are the overall statistics of the 21 equivalent configurations.

\section{MAGNETIC STRAIN NOISE}

Within a frequency domain representation, a force $F$ acting on one interferometer TM displaces it, along the laser beam propagation direction (namely, $\mathbf{z}$ ) by $\Delta L=F /\left(M \omega^{2}\right)$, where $M$ is the TM mass $(\mathrm{kg})$ and $\omega=2 \pi f$ is the frequency in $\mathrm{rad} / \mathrm{s}$. The associated strain noise in the detector is

$$
h_{\text {mag }}=\frac{\Delta L}{L_{0}},
$$

where $L_{0}=3000 \mathrm{~m}$ is the interferometer arm length.

The total $h$ due to the PAY magnetic coupling is the sum of a translational term with a rotational one. Assuming linear superposition and assuming that the magnetic forces acting on the TM are uncorrelated over a long range-so that the sum over the $4 \mathrm{TM}$ is treated as incoherent- - the term associated with the translational force is

$$
h_{\text {transl }}=\frac{1}{L_{0} M(2 \pi f)^{2}} \sqrt{\sum_{j=1}^{4}\left(\sum_{i=1}^{4} F_{i}\right)_{j}^{2}},
$$

with $M=42 \mathrm{~kg}$ being the mass of the $\operatorname{AdV} \mathrm{TM}^{2}$ and $f$ being the frequency. For each $j$ th TM, the total magnetic force is the sum of the force on the $i$ th magnet. Considering that all the magnetic moments are directed along the $\mathbf{z}$ direction and
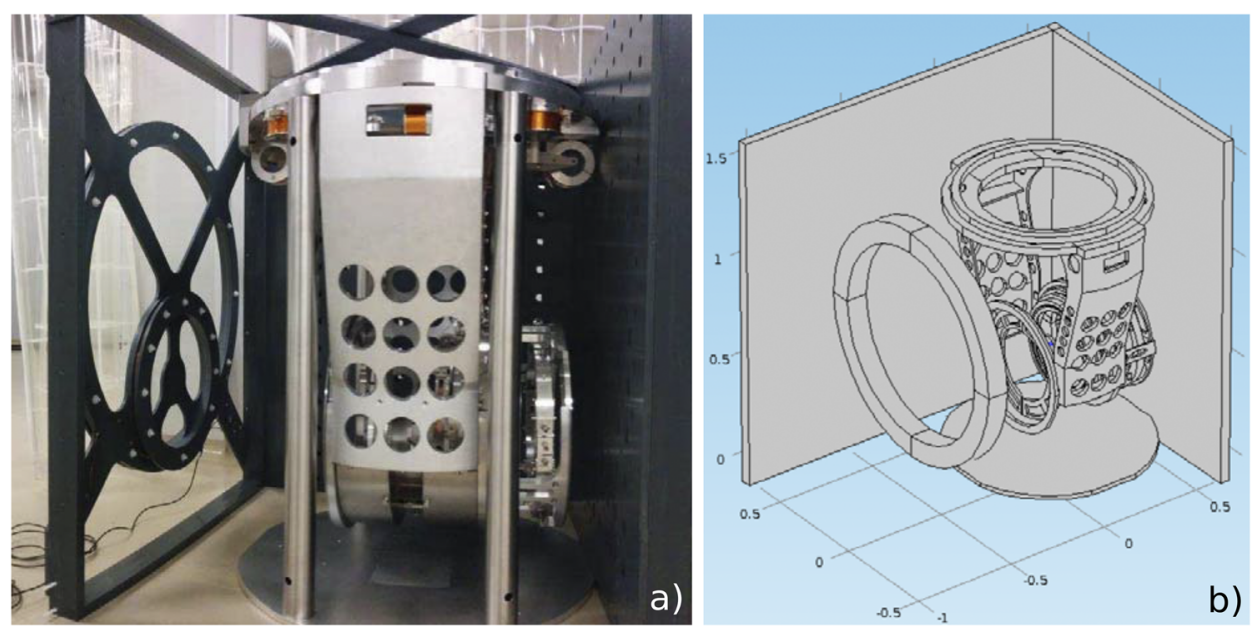

FIG. 6. The IMP inside the PVC frame: photo of the experimental apparatus (a) and the FE model (b). 
that they are constant, the translational magnetic contribution is reduced to

$$
h_{\text {transl }}=\frac{1}{L_{0} M(2 \pi f)^{2}} \sqrt{\sum_{j=1}^{4}\left[\sum_{i=1}^{4} \mu_{i}\left(\frac{\partial B_{z}}{\partial z}\right)_{i}\right]_{j}^{2}},
$$

where $B_{z}$ is the magnetic field component along $\mathbf{z}$. As the force is directed along $\mathbf{z}$, only two kind of torques exist: $\tau_{x}$ and $\tau_{y}$. Considering $F_{z}^{j}$, with $j=[1,4]$, the magnetic forces on the four magnets [as defined in Fig. 2(b)], we have

$$
\begin{aligned}
& \tau_{x}=\left[\left(F_{z}^{1}+F_{z}^{2}\right)-\left(F_{z}^{3}+F_{z}^{4}\right)\right] y, \\
& \tau_{y}=\left[\left(F_{z}^{1}+F_{z}^{4}\right)-\left(F_{z}^{2}+F_{z}^{3}\right)\right] x,
\end{aligned}
$$

where $x$ and $y$ are the components of the magnet position vector or, in other words, the force's application point relative to the TM centre mass. Hence the rotational contribution to the strain is

$$
h_{r o t}=\frac{D}{L_{0}(2 \pi f)^{2}} \sqrt{\sum_{j=1}^{4}\left(\frac{\tau_{x j}}{I_{x x}}+\frac{\tau_{y j}}{I_{y y}}\right)^{2}},
$$

where $I_{x x}$ and $I_{y y}$ are the moments of inertia of the TM and $D$ is the laser misalignment from the centre of mass of the TM (assumed the same for all TMs). Finally we can compute the total magnetic strain noise, considering the same contribution on each TM,

$$
h_{\text {mag }}=h_{\text {transl }}+h_{\text {rot }}=\frac{2 F}{M L_{0} \omega^{2}}+\frac{2 \sqrt{2} D}{I L_{0} \omega^{2}}\left(\tau_{x}+\tau_{y}\right),
$$

with $F, \tau_{x}$, and $\tau_{y}$, respectively, being the total force and torques calculated on each TM. We also assumed identical inertia moments $\left(I_{x x}=I_{y y}=I\right)$ and a conservative beam off-centering of $D=1 \mathrm{~mm}$.

\section{A. Environmental magnetic field measurement}

We based our estimation on a set of measurements carried out in August 2017. We sampled the magnetic field at several locations inside the three experimental Virgo buildings: the Central Building (CEB), where the two input TMs are located, and the two end buildings (North End Building-NEB and West End Building-WEB), which host the two end TMs. For each building, we computed the magnitude of the magnetic field vector (Fig. 7). Then we took into account the filtering effect produced by the steel tank that surrounds the suspension system, which effectively acts as a first order low pass filter with a cutoff frequency of $f_{0}=5 \mathrm{~Hz}$. Thus our estimate of the equivalent $B_{e n v}$ at the TMs is

$$
B_{e n v}=\frac{\sqrt{B_{C E B}^{2}+B_{C E B}^{2}+B_{N E B}^{2}+B_{W E B}^{2}}}{\sqrt{1+\left(f / f_{0}\right)^{2}}} .
$$

\section{B. Force calculation}

The FE model of the PAY was placed in a uniform magnetic field $B=1 \mathrm{~T}$ directed along the $\mathbf{x}, \mathbf{y}$, and $\mathbf{z}$ axes and with frequency range $f=[10,2000] \mathrm{Hz}$. We observed that the main contribution to the gradient came from $B_{z}$. The force on each magnet is computed according to Sec. III, and the net total force is the mean value of a Monte Carlo procedure to take

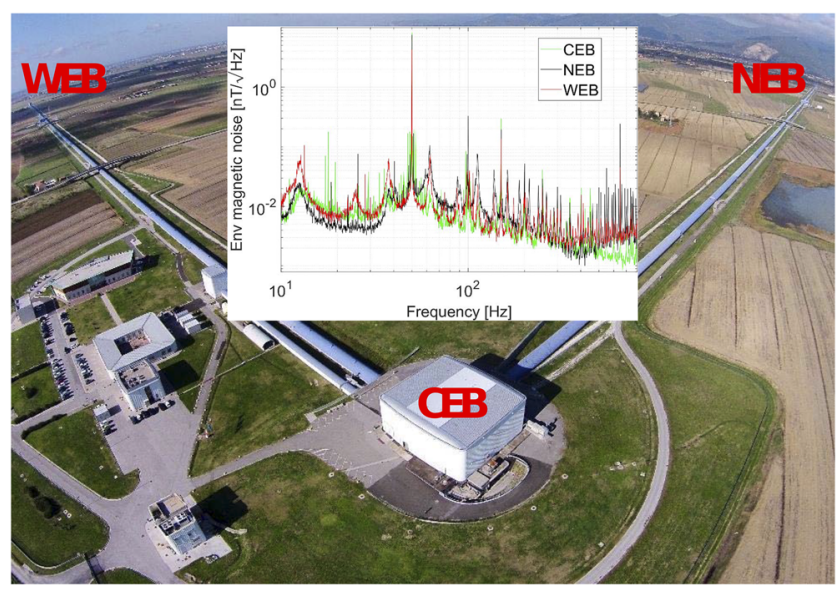

FIG. 7. Aerial photo of the Virgo site. In the inset, the environmental magnetic field activity spectrum, acquired in the three main buildings (CEB = central, $\mathrm{WEB}=$ west end, $\mathrm{NEB}=$ north end).

into account deviation from the nominal parameters both in the magnet moments and in the magnet positioning (details in the supplementary materials; Secs. III-V). This procedure was iterated for each of the 21 electrical configurations.

\section{RESULTS}

In Fig. 8(a), we show the estimation of the magnetic contribution to the AdV strain noise, calculated by multiplying $h_{\text {magn }}$ in Eq. (8) by the real magnetic field spectrum of Eq. (9). The force values used in this plot are an average on the 21 equivalent electrical configurations.

The translational force contribution (blue line) is dominant on both rotational ones (red and orange lines). The comparison with the projected sensitivity curves shows that magnetic noise (translational plus rotational) could be considered a nuisance only for the latest interferometer specifications (design - gray curve). In general though, the magnetic contribution stays well below the safety requirements (one order of magnitude below the best sensitivity limit).

When we consider the uncertainty due to the electrical configurations, we see that the spread in the calculated force values is well within safe limits. Figure 8(b) shows the envelope region due to slightly different force values computed on the 21 electrical configurations. In this plot, we also consider the combined contribution of both translational and torque forces.

Naturally, the upper expected noise gets closer to the sensitivity curves although it is still of moderate concern for the latest design specification only.

\section{DISCUSSION}

The validation step had the goal to define the electric configuration of the PAY, and the agreement between the simulated and the measured field was found to be within $5 \%$. This value is the current limit of the FE model in representing the real PAY, and it is due to all the uncertainties and simplifications embodied both in simulations and in the experimental step. 

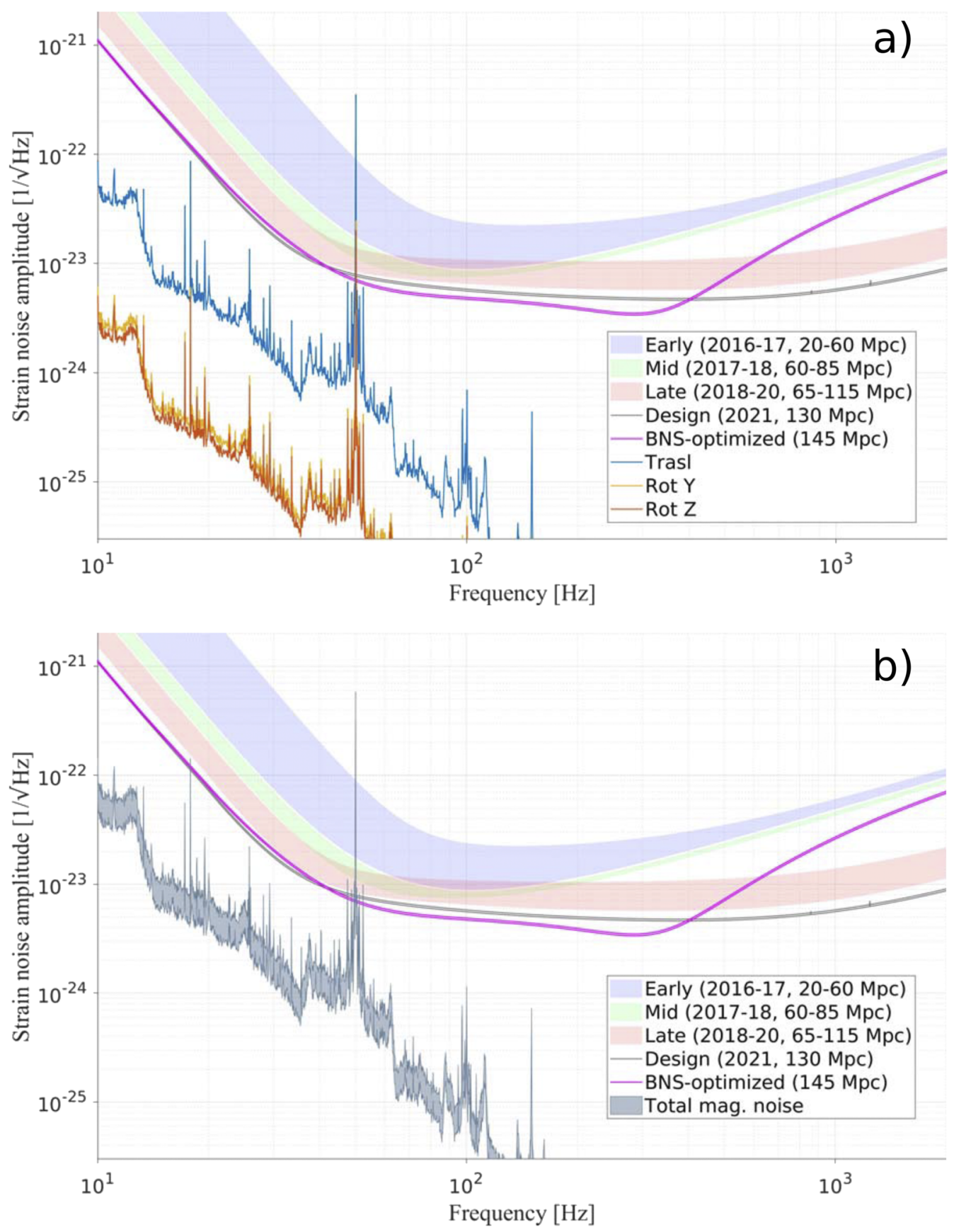

FIG. 8. Contribution of the magnetic noise to the AdV sensitivity, in the frequency range of interest for astrophysical binary merger detection. (a) Spectra of both translational (blue line) and rotational (red and orange lines) magnetic effects on the TMs; (b) total spectra envelope (translational plus rotational) on all 21 electrical configurations.

The hierarchical and simplification steps are a reasonable heuristic approach to a problem with this complexity. Yet, these steps could have introduced further uncertainties and hence the need for further refinement and study of different decompositions. Moreover, the steel and titanium parts of the PAY were neglected in the simulation, given that their contribution is suppressed by a factor of $\approx 20-30$ due to the electrical conductivity.

Forces and displacements were calculated supposing a uniform environmental magnetic field in the area around the PAY. This assumption is almost surely not verified in the real conditions. The only practical way to test the simulation forecasts is to measure the transfer function during $\mathrm{AdV}$ working conditions (i.e., during the commissioning phase).

The displacement produced by the force on the TMs summarizes all the forces acting on the four TMs of the two Fabry-Perot cavities. We assumed identical PAY structures and uncorrelated contributions so that the total displacement is calculated in quadrature. These assumptions stem from the close similarity among the IMP and the EMP (the main difference is in the compensation plate, which is present only in the IMP) and the significant distance between them. That said, the TMs in the central building are rather close so that correlation in the magnetic response cannot be excluded. This issue 
is not considered here and needs further testing. Moreover we are going to address the Schumann resonance common-mode contribution in a separate study, which lays its foundation on the paper. ${ }^{11}$

Results show that the magnetic noise budget should not impact the AdV initial observations (2017-2019). Nevertheless, in a couple of small frequency ranges, the estimated noise is higher than the desired level (technical noises are required to be $\leq 0.1$ of the incoherent sum of all fundamental noises). If these simulations are confirmed by the experimental data on the interferometer, this would prompt the drafting of mitigation strategies. For instance, the environmental field was already addressed by past mitigation actions carried out on initial Virgo (2009). These included the size reduction of TM magnets and power cable routing optimization.

As a direct consequence of this work, the design of the PAY structure was optimized to reduce gradients and shorten eddy current paths.

The agreement between the simulation and the measurement underlines the importance of an extensive validation phase; this would imply a more realistic modeling of the PAY environment and measurements on the interferometer with dedicated magnetic injections. We would therefore have a detailed comprehension of local and environmental magnetic interaction which could be used to plan more effective mitigation strategies, if needed.

\section{CONCLUSIONS}

The sensitivity of the upgraded gravitational interferometer advanced Virgo is limited by different kinds of noises. In the low frequency range, we focused on the noise associated with the coupling between the environmental magnetic field and the PAYs. Any time-varying magnetic field interacts with the metallic structure of the PAY to produce local gradients, which exert forces on the four magnets glued on the TM. These can induce worsening of the intrinsic displacement noise of the detector and, in the presence of high magnetic transients, create a glitch, which can be misinterpreted as a GW signal.

In order to understand the contribution of the magnetic noise, we studied the magnetic response of the PAY to a given environmental field. Since a direct measurement of the induced field is impractical, we had to use FE simulations.

Several steps were performed on the system in order to reduce the solution time and simplify simulation. Then, an optimization procedure based on Design of Experiment (DoE) techniques was developed to find the optimal electric configuration of the PAY.

The magnetic field gradient was calculated taking into account the magnetic moment and magnet position dishomogeneities. Monte Carlo simulations were used to compute the total force on the TM as a function of the magnetic moment tolerance and of the magnet position error, with respect to the PAY structure.

The strain noise contribution was estimated for both translational and rotational forces, and it was compared with the AdV sensitivity curve. Results suggest that the magnetic noise contribution to the strain is not dramatic for the time being, while it will possibly be an issue when the detector will approach its final stage design sensitivity and beyond it. For this reason, we are already working on performing further measurements to refine the analysis and compare it to the measurements in the actual AdV working conditions.

\section{SUPPLEMENTARY MATERIAL}

See supplementary material for further details on the system modeling, the experimental setup, and the magnetic force computation.

\section{ACKNOWLEDGMENTS}

The authors gratefully acknowledge the European Gravitational Observatory (EGO) and the Virgo Collaboration for providing access to the Central Building clean room and to the environmental data. A.C. and M.N. were supported by the INFN Doctoral Fellowship at the University of Genova.

${ }^{1}$ F. Acernese et al., "Advanced Virgo: A second-generation interferometric gravitational wave detector," Classical Quantum Gravity 32(2), 024001 (2015).

${ }^{2}$ Virgo Collaboration, "Advanced Virgo technical design report," Technical Report VIR-0128A-12, 2012.

${ }^{3}$ B. P. Abbott $e t$ al., "Observation of gravitational waves from a binary black hole merger," Phys. Rev. Lett. 116(6), 061102 (2016).

${ }^{4}$ J. Aasi et al., "Advanced LIGO," Classical Quantum Gravity 32(7), 074001 (2015).

${ }^{5}$ B. P. Abbott et al., "GW170814: A three-detector observation of gravitational waves from a binary black hole coalescence," Phys. Rev. Lett. 119(14), 141101 (2017).

${ }^{6}$ B. P. Abbott et al., "GW170817: Observation of gravitational waves from a binary neutron star inspiral," Phys. Rev. Lett. 119(16), 161101 (2017).

${ }^{7}$ LIGO-Virgo Collaboration and Others, "Multi-messenger observations of a binary neutron star merger," Astrophys. J. 848(2), L12 (2017).

${ }^{8} \mathrm{~F}$. Acernese et al., "Status of the advanced Virgo gravitational wave detector," Int. J. Mod. Phys. A 32(28n29), 1744003 (2017).

${ }^{9} \mathrm{~J}$. Aasi et al., "The characterization of Virgo data and its impact on gravitational-wave searches," Classical Quantum Gravity 29(15), 155002 (2012).

${ }^{10}$ E. Thrane, N. Christensen, and R. M. S. Schofield, "Correlated magnetic noise in global networks of gravitational-wave detectors: Observations and implications," Phys. Rev. D 87(12), 123009 (2013).

${ }^{11}$ M. W. Coughlin, A. Cirone, P. Meyers et al., "Measurement and subtraction of Schumann resonances at gravitational-wave interferometers," Phys. Rev. D 97(10), 102007 (2018).

${ }^{12}$ I. Kowalska-Leszczynska et al., "Globally coherent short duration magnetic field transients and their effect on ground based gravitational-wave detectors," Classical Quantum Gravity 34(7), 074002 (2017).

${ }^{13}$ I. Fiori, P. Ruggi, B. L. Swinkels, A. Chincarini, S. Farinon, M. Neri, P. Rapagnani, and P. Puppo, "Tentative upper limit of magnetic field distortion factor at AdV mirror actuation magnets," Virgo Technical Document VIR-0171A-14, 2014.

${ }^{14}$ A. Bernardini, E. Majorana, P. Puppo, P. Rapagnani, F. Ricci, and G. Testi, "Suspension last stages for the mirrors of the Virgo interferometric gravitational wave antenna," Rev. Sci. Instrum. 70(8), 3463-3472 (1999).

${ }^{15} \mathrm{G}$. Ballardin et al., "Measurement of the VIRGO superattenuator performance for seismic noise suppression," Rev. Sci. Instrum. 72(9), 3643-3652 (2001).

${ }^{16}$ W. B. J. Zimmerman, Multiphysics Modeling with Finite Element Methods (World Scientific Publishing Company, 2006).

${ }^{17}$ R. Holm, Electric Contacts (Springer Berlin Heidelberg, Berlin, Heidelberg, 1967).

${ }^{18}$ M. Braunovic, N. K. Myshkin, and V. V. Konchits, Electrical Contacts: Fundamentals, Applications and Technology (CRC Press, 2007). 